\title{
SELEÇÃO DE FONTES ALTERNATIVAS DE GERAÇÃO DISTRIBUÍDA UTILIZANDO UMA ANÁLISE MULTICRITERIAL BASEADA NO MÉTODO AHP E NA LÓGICA FUZZY
}

\author{
Alexandre Barin* \\ Luciane Neves Canha* \\ Karine Faverzani Magnago* \\ Alzenira da Rosa Abaide* \\ *UNIVERSIDADE FEDERAL DE SANTA MARIA - UFSM/PPGEE/CEEMA \\ Avenida Roraima, ${ }^{\circ} 1000$, Cidade Universitária, \\ Bairro Camobi, Santa Maria - RS - CEP: 97105-900.
}

\begin{abstract}
Selection of Alternative Energy Sources by Using a Multicriteria Analysis for Distributed Generation System Management: The AHP Method and the Fuzzy Logic.

This paper presents a methodology for renewable energy sources selection according to the evaluation of the main operational characteristics related with social, economic and environmental aspects. Accordingly, the proposed methodology taking as basis the theory developed by Saaty in the AHP method and multi-rules-based decision and multi-sets considerations applied in the fuzzy logic. By exploiting a multi-criteria decision making, this methodology evaluates the most important energy technologies that make use of renewable primary resources, such as - wind generators, microturbines, photovoltaic cells and fuel cells. For the purpose of the study, these technologies are evaluated with the intention to find the most appropriate renewable energy system for different scenarios, according to the selected criteria, namely: efficiency, costs, technical maturity, environmental impacts, power application range and lifecycle. In conclusion, it is presented a comparison of the final results - renewable energy sources ranking - achieved by

Artigo submetido em 17/10/2009 (Id.: 01065)

Revisado em 07/12/2009, 15/02/2010, 14/04/2010

Aceito sob recomendação do Editor Associado Prof. Ivan Nunes Da Silva
\end{abstract}

the fuzzy logic and the AHP method for environment impacts and costs scenarios.

KEYWORDS: Analytic hierarchy process (AHP), distributed generation, fuzzy logic,multicriteria analysis, renewable energy sources.

\section{RESUMO}

Este artigo apresenta uma metodologia para tomada de decisão multicriterial que visa à seleção de sistemas renováveis de energia considerando as características fundamentais de operação e aplicação destes sistemas. Esta metodologia toma como base o método Analytic Hierarchy Process (AHP) e a lógica fuzzy com intuito de avaliar os principais sistemas de geração distribuída que podem fazer uso de fontes primárias renováveis, tais como: painéis fotovoltaicos, aerogeradores, microturbinas e células de combustível com uso de biogás. Para avaliação destas fontes consideram-se os seguintes critérios: eficiência, custos, maturidade tecnológica, impactos ambientais, amplitude de atuação do sistema e vida útil. Por fim é apresentada uma classificação em ordem de prioridade para o uso das fontes de geração comparando resultados provenientes da aplicação do método AHP e da lógica fuzzy para os cenários custos e meio ambiente.

PALAVRAS-CHAVE: Análise multicriterial, fontes re- 
nováveis de energia, geração distribuída, lógica fuzzy,método AHP.

\section{INTRODUÇÃO}

Avaliando o gradativo aumento na participação de fontes alternativas e renováveis de energia na matriz energética mundial e o atual contexto do setor elétrico brasileiro, com necessidade premente de aumento na oferta de energia elétrica, percebe-se que a progressiva participação da geração distribuída (GD) nos setores de distribuição e transmissão é uma possibilidade cada vez mais efetiva (Cormio et al, 2003). A inserção da geração distribuída decorrente de fontes primárias renováveis apresenta-se, cada vez mais, como uma alternativa estratégica para o desempenho otimizado dos segmentos de distribuição e transmissão de energia, sobretudo visando à sustentabilidade na geração de energia elétrica (Farret e Simões, 2006). Em se tratando do meio ambiente, as fontes renováveis de energia reduzem emissão de gases poluentes e, conseqüentemente, colaboram para redução do efeito estufa (Hinrichs e Kleinbach, 2009). Além disso, as tecnologias relativas às fontes renováveis são ecologicamente amigáveis e contam atualmente com incentivos governamentais para a sua difusão (PROINFA, Lei 10.438/2002).

Vários artigos citam o crescimento da forte tendência na substituição da energia de origem fóssil por fontes de energia renováveis, tendo como objetivo, além da busca por novas fontes de energia, minimizar os efeitos negativos causados ao meio ambiente pelo uso excessivo dos combustíveis fósseis (Hashem, 2006), (Bernal-Agustin e Dufo-López, 2006), (Coelho et al, 2006), (Dutra e Szklo, 2008). A busca pelo desenvolvimento sustentável, em âmbitos sociais e ambientais, é um fator de extrema importância que incentiva a elaboração de várias pesquisas e projetos, investigando mecanismos de seleção e gerenciamento para o adequado uso das formas alternativas e principalmente renováveis de geração de energia.

Neste artigo será possível verificar a importância da adequada análise das diversas características associadas a um sistema de geração de energia, composto de várias formas de geração alternativa, na obtenção da máxima eficiência no uso de fontes de gerações distribuídas. A aplicabilidade da metodologia proposta pode-se dar não apenas nas situações em que os especialistas devem decidir e justificar qual o melhor tipo de geração distribuída a ser utilizado em um determinado local ou instalação, mas também nos casos em que uma rede inteligente (smart grid) poderá decidir a ordem de despacho das várias fontes de geração alternativa que compõem um sistema de geração distribuída de forma a obter a máxima eficiência operativa. Para se obter a devida classificação para a utilização destes sistemas de geração, vários trabalhos têm feito uso de diversos métodos de análise multicriterial como lógica fuzzy (Borges e Antunes, 2003), Promethee (Haralambopoulos e Polatidis, 2003), Electre (Beccali et al, 2003), Macbeth (Cvansnick, 1995 ) e AHP (Wedley et al, 2001).

Este estudo irá apresentar uma metodologia de tomada de decisão multicriterial baseada na teoria desenvolvida por Saaty, durante a criação do método AHP, e na viabilidade da modelagem matemática de variáveis subjetivas ou incertas por meio do uso de regras e conjuntos fuzzy. Em relação à escolha pelo uso de técnicas multicriteriais, deve-se observar que há uma série de parâmetros a serem analisados quando se deseja gerenciar sistemas de geração alternativos ou renováveis. Muitas vezes os interesses são conflitantes e as necessidades a serem atendidas muito diversas e, desta forma, projetos que priorizam o uso de fontes renováveis acabam sendo julgados apenas por uma única ótica, nem sempre favorável. Análises monocriteriais, que regem grande parte dos atuais projetos de geração, podem ser substituídas por análises multicriteriais que permitam a avaliação e a satisfação de diversos critérios. Embora a melhor solução não seja atingida para um parâmetro específico, ao final atinge-se uma solução harmoniosa que tende a equilibrar resultados positivos e negativos em prol de um gerenciamento mais eficiente. A aplicação prática destes processos de tomada de decisão poderá auxiliar nos sistemas inteligentes de atendimento às cargas que incorporam a preocupação com as questões econômicas, sociais e ambientais.

A escolha pela lógica fuzzy e método AHP dentre as demais opções de análises multicriteriais é justificada pelo acesso facilitado a base teórica de cada método, assim como a possibilidade de avaliação de cada passo desenvolvido durante as simulações, o que permite a corroboração dos resultados finais e, consequentemente, propicia uma elevação do grau de confiabilidade dos mesmos. Os resultados provenientes da aplicação do método AHP e da lógica fuzzy serão comparados para os cenários custos e meio ambiente com o objetivo de validar o uso da metodologia apresentada neste artigo. Ainda, visando este mesmo objetivo, as considerações descritas em (Bana e Vansnick, 2008) sobre o uso do método AHP serão ponderadas.

A partir da aplicação da metodologia sugerida este trabalho irá avaliar as potencialidades de uso dos principais sistemas de geração distribuída que podem utilizar fontes primárias renováveis, tais como: painéis fotovoltaicos, aerogeradores e microturbinas e células de combus- 
tível com uso do biogás. Para o desenvolvimento da metodologia multicriterial serão apresentados os principais parâmetros que poderão compor as análises para classificação de sistemas com diversos tipos de fontes de GD. O objetivo é obter uma classificação que indique a melhor forma de participação das fontes renováveis para se obter um gerenciamento sustentável, considerando sistemas de geração distribuída de energia. Deste modo, serão analisados os parâmetros eficiência, custos, maturidade tecnológica, impactos ambientais, amplitude de atuação do sistema (faixa de potência) e vida útil. Os parâmetros (critérios) definidos neste artigo serão identificados separadamente como qualitativos e quantitativos, sendo priorizados da forma mais adequada de acordo com os cenários custos e meio ambiente.

Deve-se observar que a metodologia desenvolvida neste artigo pode considerar diversos tipos de fontes de energia e quaisquer parâmetros operacionais referentes às mesmas, desde que seja possível o acesso ao uso de uma base de dados coesa.

A diferenciação para cada estudo de caso se dá no desenvolvimento da base de dados para análise e na ordem de prioridade dos critérios em questão. A partir destas definições, estabelecidas de acordo com as necessidades de gerenciamento e condições político-econômicas de cada região (realidade local), procede-se com a configuração dos limites e relações da metodologia multicriterial proposta.

\section{ASPECTOS PRINCIPAIS DO MÉTODO MULTICRITERIAL ADOTADO}

Para o desenvolvimento da metodologia proposta neste artigo, quatro tipos de fontes renováveis de energia foram analisados em dois diferentes cenários considerando seis diferentes parâmetros. O principal objetivo deste estudo é identificar o tipo de fonte mais apropriado a ser utilizado em cada um dos cenários em análise. Para a aplicação da metodologia, primeiramente foram definidos as fontes a serem avaliadas e os critérios a serem considerados. No passo seguinte os parâmetros selecionados foram classificados em quantitativos e qualitativos. Posteriormente, foi realizada uma classificação dos parâmetros por relevância, de acordo com os dois cenários em análise. Na seqüência, apresenta-se uma breve descrição sobre os aspectos mais importantes para o entendimento da metodologia adotada.

\subsection{Fontes Renováveis de Energia}

Este artigo considera a avaliação das principais fontes renováveis de energia que podem ser utilizadas como sistemas de geração distribuída; a saber: painéis fotovoltaicos (PV), aerogeradores (AERO), microturbinas com uso do biogás proveniente da biomassa (MT) e células de combustível com uso do biogás ou hidrogênio (FC).

\subsection{Parâmetros Quantitativos}

Os parâmetros quantitativos são representados por valores reais, ou seja, dados reais de cada característica técnica e econômica avaliada para cada fonte de geração conforme o estudo de caso em questão. Neste artigo foram analisados os seguintes parâmetros quantitativos: eficiência (EF) em \%; custo de produção de energia em US\$/MWh; vida útil (VID) em anos.

\subsection{Parâmetros Qualitativos}

Parâmetros qualitativos são aqueles que não podem ser quantificados ou possuem dificuldade de quantificação. Cada estudo de caso exige um grupo de especialistas do campo de estudo em questão. No estudo proposto no artigo, o grupo de especialistas pertence ao Centro de Estudos em Energia e Meio Ambiente da Universidade Federal de Santa Maria, sendo este grupo formado por alunos de doutorado e professores. A análise dos parâmetros qualitativos toma como base as notas estipuladas pelos especialistas, considerando as principais características dos sistemas em questão pesquisadas em fontes bibliográficas confiáveis (Farret e Simões, 2006), (Edinger e Sanjay, 2009), (Lora e Haddad, 2006), (Soares, 2007). Para a definição das notas finais de forma quantificada, tomando como base uma escala de 0 a 1 onde 1 significa maior relevância, primeiramente os especialistas atribuem as notas individualmente. Em um segundo momento as notas são discutidas entre os especialistas com intuito de eliminar, ou reduzir, discordâncias muito significativas entre as notas dadas por diferentes especialistas para um mesmo parâmetro.

É importante salientar que estas notas devem ser definidas por tomadores de decisão experientes e sempre considerando uma base de dados atual. Mesmo assim, em alguns casos estas notas podem ser consideradas subjetivas, havendo a possibilidade de discordâncias entre as notas estipuladas por diferentes especialistas. A eliminação destas discordâncias é uma etapa fundamental para o não comprometimento dos resultados finais, ou seja, as notas devem ser discutidas entre os especialistas. 
Apenas depois desta discussão, deve-se realizar a média das notas parciais de cada especialista, resultando nas notas finais. Ainda em relação a definição das notas, a análise dos especialistas deve ser concebida não apenas de acordo com dados baseados em fontes bibliográficas confiáveis e atuais, mas também considerando a realidade local, avaliando as condições econômicas, sociais e ambientais de região onde prevê-se a instalação do sistema de geração.

Neste artigo foram analisados os seguintes parâmetros qualitativos: maturidade tecnológica (MT); impactos ambientais (IA) relacionados com impactos visual e biológico, presença de elementos tóxicos e emissões de gases poluentes; amplitude de atuação do sistema (FP), considerando a faixa de potência usual da aplicação do sistema.

\subsection{Classificação dos Parâmetros por Rele- vância: Cenários Custose Meio Ambi- ente}

Nos cenários avaliados neste estudo, considera-se previamente uma classificação de relevância dos parâmetros com a relação às características dos mesmos para cada cenário em questão. Esta classificação permanece inalterada durante todo o processo de simulação da metodologia, sendo utilizada no método AHP e no uso da lógica fuzzy. O principal objetivo desta classificação é facilitar o desenvolvimento das simulações, assim como o entendimento dos especialistas sobre os passos seguidos na metodologia proposta. Deve-se observar que a construção destas classificações deve ser baseada na experiência de especialistas considerando o conjunto de parâmetros para cada cenário em questão. A relevância dos parâmetros definida para cada cenário está apresentada abaixo:

- cenário custos: $1^{\circ}$ custos, $2^{\circ}$ maturidade tecnológica e eficiência, $3^{\circ}$ vida útil, $4^{\circ}$ amplitude de atuação do sistema e $5^{\circ}$ impactos ambientais.

- cenário meio ambiente: $1^{\circ}$ impactos ambientais, $2^{\circ}$ eficiência, e vida útil, $3^{\circ}$ maturidade tecnológica, $4^{\circ}$ amplitude de atuação do sistema e $5^{\circ}$ custos.

\subsection{Base de Dados}

A base de dados utilizada para o desenvolvimento da metodologia proposta é apresentada na Tabela 1 (Farret e Simões, 2006), (Edinger e Sanjay, 2009), (Lora e Haddad, 2006), (Cobas, 2006), (Allison e Lents, 2006), (Soares, 2007), considerando os valores reais para a análise dos parâmetros quantitativos e os pesos atribuídos aos parâmetros qualitativos.

Tabela 1: Base de dados utilizada no método AHP e na lógica fuzzy

\begin{tabular}{|l|r|r|r|r|}
\hline Parâmetros & PV & AERO & MT & FC \\
\hline \hline Quantitativos & \multicolumn{5}{|c|}{ Valores reais } \\
\hline EF - Eficiência (\%) & 15 & 30 & 35 & 45 \\
\hline Custos (US\$ / MWh) & 900 & 150 & 200 & 300 \\
\hline VID - Vida útil (anos) & 28 & 25 & 20 & 8 \\
\hline Qualitativos & \multicolumn{7}{|c|}{ Pesos "quanto maior, melhor" } \\
\hline MT - Maturidade tecnológica & 0,50 & 0,80 & 0,60 & 0,70 \\
\hline IA - Impactos ambientais & 0,75 & 0,85 & 0,40 & 0,45 \\
\hline FP - Amplitude de atuação & 0,50 & 1,00 & 0,40 & 0,80 \\
\hline
\end{tabular}

\section{ANÁLISE MULTICRITERIAL: AHP}

Uma das principais características da metodologia desenvolvida por Saaty no método AHP (Saaty, 1991) é a relação entre o processo de tomada de decisão desenvolvido no método e o raciocínio humano. Segundo Saaty, a mente humana distribui elementos em grupos de acordo com propriedades, estruturando o raciocínio e a conseqüente tomada de decisão. No uso do AHP, estes agrupamentos podem ser descritos como hierarquias. No método de Saaty, a partir da formulação destas hierarquias há o estabelecimento de importâncias (pesos), normalmente determinados através de julgamentos verbais e numéricos. $\mathrm{O}$ valor atribuído aos pesos segue expressões definidas por Saaty, descritas na Tabela 2.

Tabela 2: Expressões trazidas por Saaty

\begin{tabular}{|l|l|l|}
\hline $\begin{array}{l}\text { Intensidade } \\
\text { da } \\
\text { Importância }\end{array}$ & $\begin{array}{l}\text { Definição } \\
\text { (termos verbais) }\end{array}$ & Explicação \\
\hline \hline 1 & $\begin{array}{l}\text { Igualmente impor- } \\
\text { tante. }\end{array}$ & $\begin{array}{l}\text { Dois elementos contribuem } \\
\text { igualmente. }\end{array}$ \\
\hline 3 & $\begin{array}{l}\text { Moderadamente } \\
\text { mais importante. }\end{array}$ & $\begin{array}{l}\text { Experiência e julgamento fa- } \\
\text { vorecem ligeiramente um ele- } \\
\text { mento. }\end{array}$ \\
\hline 5 & $\begin{array}{l}\text { Fortemente mais im- } \\
\text { portante. }\end{array}$ & $\begin{array}{l}\text { Experiência e julgamento fa- } \\
\text { vorecem fortemente um ele- } \\
\text { mento. }\end{array}$ \\
\hline 7 & $\begin{array}{l}\text { Muito fortemente } \\
\text { mais importante } \\
\text { ou importância } \\
\text { confirmada. }\end{array}$ & $\begin{array}{l}\text { Elemento fortemente favore- } \\
\text { cido. A dominância é pro- } \\
\text { vada na prática. }\end{array}$ \\
\hline 9 & $\begin{array}{l}\text { Extremamente mais } \\
\text { importante. }\end{array}$ & $\begin{array}{l}\text { A evidência favorece um ele- } \\
\text { mento em relação a outro na } \\
\text { ordemmais alta. }\end{array}$ \\
\hline
\end{tabular}

Estes pesos ou graus de importância são estabelecidos em um processo de comparação por pares organizados em matrizes de comparações paritárias (MCP). Os valores da MCP representam a escolha da alternativa da linha versus a alternativa da coluna. Esta comparação por pares permite avaliar a consistência da decisão para cada nível da hierarquia. Tomando como base os dados apresentados na Tabela 1 e as expressões trazidas por Saaty na Tabela 2, fez-se uso do método AHP avaliando os parâmetros selecionados com intuito de encon- 
trar a melhor forma de geração distribuída renovável de energia. Para o correto desenvolvimento das MCPs, Saaty propõe ainda o cálculo de um indicador de inconsistência dos julgamentos, denominada Razão de Consistência (C.R. - Consistency Ratio). Para o cálculo de C.R. o AHP faz uso do índice de consistência (C.I.) que evita que as comparações (julgamentos de prioridades) que possuem altos níveis de inconsistência sejam aceitas. Com o objetivo de medir o desvio de consistência, usa-se a seguinte fórmula:

$$
C . I=\frac{\lambda_{\max }-n}{n-1}
$$

onde: $\quad \lambda_{\max }-n$ é o desvio dos julgamentos em relação a consistência e $n$ é o valor que representa a ordem da matriz.

Seguindo as orientações de Saaty, deve-se verificar a coerência dos julgamentos com a análise do autovalor $\lambda$, da matriz de julgamentos (MCP). Caso todos os julgamentos sejam coerentes entre si, o autovalor máximo $\lambda_{\max }$ será igual à ordem da matriz. Segundo Saaty, para matrizes recíprocas positivas, $\lambda_{\max }$ pode ser estimado como o produto de um vetor composto pela soma das colunas da matriz de julgamentos pelo autovetor. Por incorporarem a subjetividade da complexidade do julgamento humano, a cada passo é necessário verificar a validade dos resultados. De uma forma mais clara, pode-se descrever que os valores de $\lambda_{\max }$ de cada MCP são calculados da seguinte forma:

- somam-se os valores de cada coluna da MCP;

- o valor de cada uma destas somas é multiplicado pelo valor do peso relativo encontrado correspondente a alternativa fundamental (dentro da diagonal principal da MCP) de cada coluna em questão;

- os valores destas multiplicações são somados resultando no valor de $\lambda_{\max }$ para esta matriz.

A partir destas etapas obtém-se $\lambda_{\max }$ e se faz uso do valor da ordem da matriz $n$ para o cálculo de C.I.. Para o cálculo final de C.R. um índice aleatório (R.I.) na ordem de $n$ elementos é calculado como média do C.I. para várias matrizes geradas na escala de 1 a 9 , com reciprocidade forçada. Os valores de R.I. das matrizes estão descritos em (Saaty, 1991). Por fim, C.R. pode ser encontrado pela razão definida por C.I sobre R.I..

Para um melhor entendimento da aplicação da análise multicriterial através do do uso do método AHP, estruturou-se o desenvovimento do método em três processos. Na construção da MCP das alternativas (Processo 1 - Tabela 3 ), calculam-se as prioridades relativas (RW) entre as alternativas considerando separadamente cada um dos parâmetros. De forma simplificada, RW resulta da divisão de cada valor da MCP pela soma dos valores da coluna onde esse elemento se encontra e, posteriormente, calcula-se a média para cada linha. $\mathrm{Na}$ construção da MCP dos parâmetros (Processo 2 - Tabelas 4 e 5), calculam-se as prioridades relativas apenas entre os parâmetros, para cada cenário. A aplicação matemática procede-se da mesma forma anterior. Com objetivo de corroborar os resultados provenientes das MCPs, calcula-se a razão de consistência (C.R.) de cada MCP. Segundo Saaty o valor calculado para C.R. não deve ser maior que $10 \%(0,1)$ para matrizes com cinco ou mais elementos.

Na última etapa, multiplicam-se os valores das ponderações (RW) das alternativas (Processo 1) pela ponderação obtida de cada critério (Processo 2). Estas multiplicações originam uma última matriz de mesma ordem da primeira. Os valores de cada linha desta matriz são somados resultando nas prioridades relativas finais (FRW) que definem a classificação das alternativas para os cenários custos e meio ambiente (Processo 3 - Tabelas 6 e 7).

As Tabelas 6 e 7 apresentam as prioridades relativas finais (FRW) calculadas e a classificação final (CL) das fontes renováveis de GD considerando os cenários meio ambiente e custos, respectivamente.

\section{ANÁLISE MULTICRITERIAL: LÓGICA FUZZY}

Resumidamente, a lógica fuzzy é uma técnica inteligente que faz uso da representação da forma humana de pensar, simulando a habilidade de tomar decisões em ambientes imprecisos, permitindo lidar com problemas de natureza incerta ou nebulosa (Shaw e Simões, 1999). Uma aplicação da lógica

fuzzy é a construção de sistemas fuzzy, que são sistemas especialistas compostos da análise de parâmetros quantitativos e qualitativos (entrada), método de fuzzificação e modelagem, regras fuzzy, inferência fuzzy (avaliação das regras e agregação), método de defuzzificação e classificação final (saída numérica devidamente classificada de forma linguística).

Para processar as entradas do sistema, realiza-se primeiramente a fuzzificação, que consiste no emprego das funções de pertinência para traduzir cada um dos va- 
Tabela 3: Determinação dos pesos entre as alternativas - (Processo 1 )

\begin{tabular}{|c|c|c|c|c|c|}
\hline \multicolumn{6}{|c|}{ Parâmetro 1 - Eficiência $(\mathrm{CR}=0,0811)$} \\
\hline & $\mathrm{PV}$ & AERO & MT & $\mathrm{FC}$ & RW \\
\hline PV & 1,00 & 0,20 & 0,20 & 0,11 & 0,04 \\
\hline AERO & 5,00 & 1,00 & 1,00 & 0,20 & 0,17 \\
\hline MT & 5,00 & 1,00 & 1,00 & 0,20 & 0,17 \\
\hline $\mathrm{FC}$ & 9,00 & 5,00 & 5,00 & 1,00 & 0,63 \\
\hline \multicolumn{6}{|c|}{ Parâmetro $2-$ Custos $(\mathrm{CR}=0,0636)$} \\
\hline & PV & AERO & MT & $\mathrm{FC}$ & RW \\
\hline PV & 1,00 & 0,11 & 0,14 & 0,14 & 0,04 \\
\hline AERO & 9,00 & 1,00 & 1,00 & 3,00 & 0,40 \\
\hline MT & 7,00 & 1,00 & 1,00 & 3,00 & 0,38 \\
\hline $\mathrm{FC}$ & 7,00 & 0,33 & 0,33 & 1,00 & 0,18 \\
\hline \multicolumn{6}{|c|}{ Parâmetro 3 - Maturidade tecnológica $(\mathrm{CR}=0,0758)$} \\
\hline & PV & AERO & MT & $\mathrm{FC}$ & RW \\
\hline PV & 1,00 & 0,20 & 0,20 & 0,20 & 0,06 \\
\hline AERO & 5,00 & 1,00 & 3,00 & 1,00 & 0,38 \\
\hline MT & 5,00 & 0,33 & 1,00 & 0,33 & 0,18 \\
\hline $\mathrm{FC}$ & 5,00 & 1,00 & 3,00 & 1,00 & 0,38 \\
\hline \multicolumn{6}{|c|}{ Parâmetro 4 - Impactos ambientais $(\mathrm{CR}=0,0661)$} \\
\hline & PV & AERO & MT & $\mathrm{FC}$ & RW \\
\hline PV & 1,00 & 0,33 & 5,00 & 3,00 & 0,26 \\
\hline AERO & 3,00 & 1,00 & 7,00 & 5,00 & 0,56 \\
\hline MT & 0,20 & 0,14 & 1,00 & 0,33 & 0,06 \\
\hline $\mathrm{FC}$ & 0,33 & 0,20 & 3,00 & 1,00 & 0,12 \\
\hline \multicolumn{6}{|c|}{ Parâmetro 5 - Faixa de potência $(\mathrm{CR}=0,0037)$} \\
\hline & PV & AERO & MT & $\mathrm{FC}$ & RW \\
\hline PV & 1,00 & 0,11 & 1,00 & 0,11 & 0,05 \\
\hline AERO & 9,00 & 1,00 & 7,00 & 1,00 & 0,43 \\
\hline MT & 1,00 & 0,14 & 1,00 & 0,11 & 0,05 \\
\hline $\mathrm{FC}$ & 9,00 & 1,00 & 9,00 & 1,00 & 0,46 \\
\hline \multicolumn{6}{|c|}{ Parâmetro 6 - Vida útil $(\mathrm{CR}=0,0956)$} \\
\hline & PV & AERO & MT & $\mathrm{FC}$ & RW \\
\hline PV & 1,00 & 3,00 & 5,00 & 9,00 & 0,56 \\
\hline AERO & 0,33 & 1,00 & 3,00 & 7,00 & 0,27 \\
\hline MT & 0,20 & 0,33 & 1,00 & 5,00 & 0,13 \\
\hline FC & 0,11 & 0,14 & 0,20 & 1,00 & 0,04 \\
\hline
\end{tabular}

lores de entrada para valores fuzzificados. Em outras palavras, nessa fase, são acionados os conjuntos fuzzy (armazenados no sistema fuzzy) responsáveis pela modelagem das variáveis de entrada, convertendo-as do formato crisp para o formato fuzzy. Desta forma, todos os valores das variáveis de entrada podem ser traduzidos de forma lingüística (por exemplo, alto, médio e baixo).

A pertinência de um elemento num conjunto fuzzy não é uma questão de afirmação ou negação, mas uma questão de grau. Este grau representa um nível de compatibilidade do elemento sobre o conjunto, na qual o valor zero significa não participação, o valor um significa participação plena e demais valores entre zero e um significam participação parcial. Os graus de pertinência são obtidos através das funções de pertinência que são representadas por formas geométricas diversas. As principais formas utilizadas são: triangular, trapezoidal e gaussiana. Os responsáveis pela modelagem destas funções devem escolher uma representação que se aproxima melhor das características dos parâmetros em questão (Cox, 1994). O número prático de funções de pertinência é algo entre 2 e 7 para cada conjunto fuzzy (que representa cada um dos parâmetros em questão).
Tabela 4: Determinação dos pesos entre critérios - cenário custos (Processo 2)

\begin{tabular}{|c|c|c|c|c|c|c|c|}
\hline \multicolumn{1}{|c|}{ MCP - Parâmetros, cenário custos (Processo 2) } \\
\hline & EF & $\$$ & MT & IA & FP & VID & RW \\
\hline EF & 1,00 & 0,20 & 1,00 & 5,00 & 3,00 & 1,00 & 0,13 \\
\hline$\$$ & 5,00 & 1,00 & 3,00 & 9,00 & 7,00 & 3,00 & 0,43 \\
\hline MT & 1,00 & 0,33 & 1,00 & 7,00 & 5,00 & 3,00 & 0,21 \\
\hline IA & 0,20 & 0,11 & 0,14 & 1,00 & 0,20 & 0,20 & 0,03 \\
\hline FP & 0,33 & 0,14 & 0,20 & 5,00 & 1,00 & 0,20 & 0,06 \\
\hline VID & 1,00 & 0,33 & 0,33 & 5,00 & 5,00 & 1,00 & 0,14 \\
\hline \multicolumn{810}{|c|}{ CR $=0,0970$} \\
\hline
\end{tabular}

Tabela 5: Determinação dos pesos entre critérios - cenário meio ambiente (Processo 2)

\begin{tabular}{|c|c|c|c|c|c|c|c|}
\hline \multicolumn{1}{|c|}{ MCP - Parâmetros, meio ambiente (Processo 2) } \\
\hline & EF & $\$$ & MT & IA & FP & VID & RW \\
\hline EF & 1,00 & 7,00 & 3,00 & 0,33 & 5,00 & 1,00 & 0,19 \\
\hline$\$$ & 0,14 & 1,00 & 0,20 & 0,11 & 0,33 & 0,14 & 0,03 \\
\hline MT & 0,33 & 5,00 & 1,00 & 0,20 & 3,00 & 0,20 & 0,09 \\
\hline IA & 3,00 & 9,00 & 5,00 & 1,00 & 9,00 & 3,00 & 0,43 \\
\hline FP & 0,20 & 3,00 & 0,33 & 0,11 & 1,00 & 0,20 & 0,05 \\
\hline VID & 1,00 & 7,00 & 5,00 & 0,33 & 5,00 & 1,00 & 0,22 \\
\hline \multicolumn{10}{|c|}{ CR $=0,0757$} \\
\hline
\end{tabular}

Tabela 6: Classificação final pelo método AHP - cenário meio ambiente (Processo 3)

\begin{tabular}{|c|c|c|c|c|c|c|c|c|}
\hline & EF & $\$$ & MT & IA & FP & VID & FRW & CL \\
\hline PV & 0,01 & 0,00 & 0,01 & 0,11 & 0,00 & 0,12 & $\mathbf{0 , 2 5 0}$ & $\mathbf{2}^{\mathbf{O}}$ \\
\hline AERO & 0,03 & 0,01 & 0,03 & 0,24 & 0,02 & 0,06 & $\mathbf{0 , 3 9 3}$ & $\mathbf{1}^{\mathbf{o}}$ \\
\hline MT & 0,03 & 0,01 & 0,02 & 0,02 & 0,00 & 0,03 & $\mathbf{0 , 1 1 4}$ & $\mathbf{4}^{\mathbf{O}}$ \\
\hline FC & 0,12 & 0,00 & 0,03 & 0,05 & 0,02 & 0,01 & $\mathbf{0 , 2 4 2}$ & $\mathbf{3}^{\text {o }}$ \\
\hline
\end{tabular}

Tabela 7: Classificação final pelo método AHP - cenário custos (Processo 3)

\begin{tabular}{|c|c|c|c|c|c|c|c|c|}
\hline & EF & $\$$ & MT & IA & FP & VID & FRW & CL \\
\hline PV & 0,01 & 0,02 & 0,01 & 0,01 & 0,00 & 0,08 & $\mathbf{0 , 1 2 5}$ & $4^{\circ}$ \\
\hline AERO & 0,02 & 0,17 & 0,08 & 0,02 & 0,03 & 0,04 & $\mathbf{0 , 3 5 3}$ & $\mathbf{1}^{\circ}$ \\
\hline MT & 0,02 & 0,16 & 0,04 & 0,00 & 0,00 & 0,02 & $\mathbf{0 , 2 4 6}$ & $\mathbf{3}^{\circ}$ \\
\hline FC & 0,08 & 0,07 & 0,08 & 0,00 & 0,03 & 0,01 & $\mathbf{0 , 2 7 6}$ & $\mathbf{2}^{\circ}$ \\
\hline
\end{tabular}

Quanto maior o número de funções, maior a precisão. No entanto, um número maior de funções de pertinência ocasiona um número maior de regras, exigindo uma demanda computacional bem mais significativa. Experiências mostraram que uma mudança de 5 funções triangulares para 7 aumenta a precisão em 15\%; a partir de valores maiores não há melhorias significativas (Shaw e Simões, 1999).

Após a fuzzificação, realiza-se a inferência fuzzy, que consiste na aplicação de regras heurísticas que relacionam as variáveis de entrada às variáveis intermediárias e estas às de saída (Shaw e Simões, 1999). A definição dessas regras constitui um aspecto fundamental no desempenho de um sistema de inferência, devendo ser realizada com auxílio do especialista. Entretanto, extraí-las de especialistas na forma de sentenças do tipo SE - EN- 
TÃO pode ser um processo adverso, com redundâncias e regras desnecessárias. O número de regras possíveis em uma simulação de um sistema fuzzy está diretamente relacionado a quantidade de variáveis linguísticas consideradas para cada parâmetro em questão. Para cada variável lingüística atribuída a um parâmetro (alto, médio, baixo) é definida uma função de pertinência dentro do conjunto fuzzy que representa este parâmetro. Quanto maior o número de variáveis lingüísticas atribuídas aos antecedentes, maior a dificuldade de se estabelecer uma base de regras consistente.

Uma forma de auxiliar o desenvolvimento das regras é a criação de uma relevância prévia dos parâmetros que estão sendo avaliados dentro do sistema fuzzy em análise (Barin, 2009). Neste processo são estabelecidas mais variáveis linguísticas aos parâmetros prioritários (mais importantes) e menos variáveis linguísticas aos parâmetros não prioritários (menos importantes). Com isso, o aumento no número de regras só acontece para os parâmetros prioritários, onde há necessidade de simulação com maior precisão considerando o estudo de caso (cenário) em questão. Ainda, de acordo com a ordem de relevância dos parâmetros, utilizam-se regras dominantes conforme o ambiente em análise. Por exemplo: para a análise de custos (cenário custos), se o custo de uma fonte de geração de energia é extremamente alto, então a saída é "inutilizável", independente das regras dos outros parâmetros existentes no sistema fuzzy. O método de relevância prévia reduz consideravelmente o número final de regras no processo de inferência fuzzy, além de facilitar o entendimento do especialista na análise do problema proposto.

Finalmente, procede-se com o processo de defuzzificação. Defuzzificação é um método utilizado para transpor as saídas do domínio fuzzy para o domínio numérico crisp, sendo esta a operação inversa da fuzzificação (Shaw e Simões, 1999). Os principais métodos de defuzzificação são: Centro-da-Área, o Centro-do-Máximo a Média-do-Máximo e a Média-Ponderada

Neste estudo, um sistema baseado em regras fuzzy foi implementado no software MATLABR, avaliando o mesmo estudo de caso descrito anteriormente para o método AHP. Os valores utilizados para os parâmetros quantitativos e os pesos definidos aos parâmetros qualitativos podem ser observados na Tabela 1 . Os conjuntos fuzzy que caracterizam cada parâmetro estão ilustrados na Figura 1, apresentada no final deste artigo. As regras desenvolvidas nesta etapa tomam como base proposições SE-ENTÃO. Fazendo uso do software MATLABß, utilizou-se o Método de Mamdani na estruturação do sistema fuzzy e o Método do Centro de Gravidade (COG) para o processo de defuzzificação (Pedrycz e Gomide, 1988). O uso do Método de Mamdani é justificado pelas seguintes características (Liang e Wang, 1991):

- pode ser utilizado em análises de engenharia, pois seus dados de entrada e saída são compostos por valores reais;

- incorpora as regras fuzzy com praticidade seguindo o raciocínio humano de análise;

- possui diversos tipos de fuzzificadores, métodos de inferência e defuzzificadores, o que atribui um maior grau de liberdade ao programador, assim como diversifica os tipos (natureza) de problemas que podem ser analisados;

- possibilita uma integração lingüística e numérica de forma consistente.

Com relação ao uso do defuzzificador COG, esta escolha é justificada pelo fato deste defuzzificador ser utilizado não apenas na análise de parâmetros qualitativos, mas também de parâmetros quantitativos. A atribuição do número de funções para cada conjunto fuzzy e a definição das regras fuzzy possuem como base a classificação dos parâmetros por relevância de acordo com os dois cenários em análise, apresentada anteriormente não seção 2 .

Ainda, o tipo de função dos conjuntos fuzzy e a largura da base de cada função foram definidos de acordo com as características de cada parâmetro em análise, apresentadas na Tabela 1.

A classificação final da utilização das fontes renováveis de energia obtida através da lógica fuzzy está apresentada na Tabela 8 para os cenários custos e meio ambiente.

Tabela 8: Classificação final pela lógica fuzzy - cenários custos e meio ambiente

\begin{tabular}{|c|c|c|c|c|}
\hline Fontes & FRW - Custos & CL & FRW - Ambiente & CL \\
\hline PV & $\mathbf{0 , 0 9 0}$ & $\mathbf{4}^{\circ}$ & $\mathbf{0 , 5 0 0}$ & $\mathbf{2}^{\mathbf{}}$ \\
\hline AERO & $\mathbf{0 , 7 5 0}$ & $\mathbf{1}^{\mathbf{O}}$ & $\mathbf{0 , 7 5 0}$ & $\mathbf{1}^{\mathbf{O}}$ \\
\hline MT & 0,517 & $\mathbf{3}^{\mathbf{O}}$ & $\mathbf{0 , 3 7 6}$ & $\mathbf{4}^{\mathbf{O}}$ \\
\hline FC & $\mathbf{0 , 6 7 6}$ & $\mathbf{2}^{\mathbf{O}}$ & $\mathbf{0 , 4 2 3}$ & $\mathbf{3}^{\mathbf{O}}$ \\
\hline
\end{tabular}

\section{COMPARAÇÃO DOS RESULTADOS: AHP E LÓGICA FUZZY}

Nas Tabelas 9 e 10 estão apresentadas as classificações finais das fontes renováveis de energia provenientes das análises multicriteriais aplicadas no método AHP e nos 
Tabela 9: Classificações finais - cenário custos

\begin{tabular}{|c|c|c|c|c|}
\hline \multirow{2}{*}{ Fontes } & \multicolumn{2}{|c|}{ AHP } & \multicolumn{2}{c|}{ Fuzzy } \\
\cline { 2 - 5 } & FRW & CL & FRW & CL \\
\hline PV & 0,125 & $\mathbf{4}^{\circ}$ & 0,090 & $4^{\mathbf{}}$ \\
\hline AERO & 0,353 & $\mathbf{1}^{\circ}$ & 0,750 & $\mathbf{1}^{\circ}$ \\
\hline MT & 0,246 & $\mathbf{3}^{\circ}$ & 0,517 & $\mathbf{3}^{\circ}$ \\
\hline FC & 0,276 & $\mathbf{2}^{\text {O }}$ & 0,676 & $\mathbf{2}^{\mathbf{O}}$ \\
\hline
\end{tabular}

Tabela 10: Classificações finais - cenário meio ambiente

\begin{tabular}{|c|c|c|c|c|}
\hline \multirow{2}{*}{ Fontes } & \multicolumn{2}{|c|}{ AHP } & \multicolumn{2}{c|}{ Fuzzy } \\
\cline { 2 - 5 } & FRW & CL & FRW & CL \\
\hline PV & 0,250 & $\mathbf{2}^{\mathbf{O}}$ & 0,500 & $\mathbf{2}^{\mathbf{O}}$ \\
\hline AERO & 0,393 & $\mathbf{1}^{\mathbf{O}}$ & 0,750 & $\mathbf{1}^{\mathbf{O}}$ \\
\hline MT & 0,114 & $\mathbf{4}^{\mathbf{O}}$ & 0,376 & $\mathbf{4}^{\mathbf{O}}$ \\
\hline FC & 0,242 & $\mathbf{3}^{\mathbf{O}}$ & 0,423 & $\mathbf{3}^{\mathbf{O}}$ \\
\hline
\end{tabular}

conjuntos e regras fuzzy, considerando os cenários custos e meio ambiente, respectivamente.

Analisando os resultados acima, percebe-se que a classificação das fontes renováveis de energia é exatamente a mesma tanto na análise com o método AHP quanto no uso da lógica fuzzy. Estes resultados corroboram a utilização destes dois métodos para a avaliação das principais características das fontes renováveis de energia. Neste caso, o sistema aerogerador é a escolha mais apropriada tanto para o cenário custos quanto para o cenário meio ambiente. Observando os valores da Tabela 1 é possível verificar que através de uma análise monocriterial o sistema aerogerador também seria selecionado como a melhor opção para os dois cenários em questão. No entanto, algumas das demais classificações, como por exemplo, a segunda posição para célula de combustível no cenário custos, contradizem os resultados de uma análise monocritério. Isto demonstra que a partir do uso de técnicas multicriteriais há uma avaliação mais ampla das características de cada estudo de caso, ponderando todos os parâmetros envolvidos.

Ainda, é essencial enfatizar que este estudo pode considerar diversos critérios e cenários simplesmente ajustando regras e relevâncias para cada caso. Isto permite a utilização do método proposto em quaisquer estudos de caso que objetivem a seleção de respostas mais adequadas a partir da análise das principais características de cada sistema em questão.

\section{CONCLUSÃO}

Este artigo apresenta um estudo visando à classificação de fontes renováveis de energia avaliando suas principais características operacionais em dois diferentes cenários: meio ambiente e custos. Para alcançar este objetivo, fez-se uso do método AHP e de conjuntos e regras fuzzy com o desenvolvimento de uma análise multicriterial uti- lizando parâmetros quantitativos e qualitativos.

Como parte da metodologia, os parâmetros foram previamente classificados de acordo com sua relevância para cada cenário em questão. Este procedimento teve como objetivo aprimorar o desenvolvimento das simulações e obter um entendimento mais claro sobre a construção da metodologia proposta. Na aplicação do método AHP, as principais considerações observadas em (Bana, 2008) foram verificadas. A partir destas, pode-se concluir que as relações entre os valores e os julgamentos foram devidamente respeitadas no estudo de caso desenvolvido. Os resultados finais corroboraram o uso do método AHP e da lógica fuzzy, assim como validaram a utilização da classificação prévia de relevância aplicada aos parâmetros.

Levando em consideração o crescente aumento da participação de fontes alternativas e renováveis de energia na matriz energética mundial e o atual contexto do setor elétrico brasileiro, é necessário reconhecer a importância da utilização de metodologias multicriteriais que avaliem da melhor forma a gestão das diversas características que compõem um sistema de geração e consumo de energia. Embora nem sempre a melhor solução seja atingida para um parâmetro específico, relacionado diretamente ao cenário em questão, ao final pode-se obter uma solução harmoniosa que tende a equilibrar resultados positivos e negativos em prol de um gerenciamento mais eficiente e confiável.

\section{AGRADECIMENTOS}

Os autores agradecem a UFSM e a CAPES.

\section{REFERÊNCIAS BIBLIOGRÁFICAS}

Allison, J.E, Lents, J. (2002). Encouraging distributed generation of power that improves air quality: can we have our cake and eat it too Power Energy, vol. 30, pp. $737-752$.

Bana E Costa, A., Vansnick, J. (2008). A critical analysis of the eigenvalue method used to derive priorities in AHP; European Journal of Operational Research, vol. 187, pp. 1422-1428.

Barin, A., Canha, L. N., Magnago, K. F., Abaide, A. R. (2009) Renewable Hybrid Systems using Biogas Fuzzy Multi-Sets and Fuzzy Multi-Rules. Energy Conversion Congress and Exposition, ECCE 2009, San José, California.

Beccali, M., Cellura, M., Mistretta, M. (2003). Decision-making in energy planning-application of 
the ELECTRE method at regional level for the diffusion of renewable energy technology; Renewable Energy, vol. 28 (13), pp. 2063-2087.

Bernal-Agustin, J. L., Dufo-López, R. (2006). Economical and environmental analysis of grid connected photovoltaic systems in Spain; Renewable Energy, vol. 31, pp. 1107-1128.

Borges, A.R., Antunes, C.H. (2003). A fuzzy multiple objective decision support model for energyeconomy planning; European Journal of Operational Research, vol. 145, pp. 304-316.

Cobas, V.R.M. (2006) Análise de sistemas híbridos com células a combustível de oxido sólido e Microturbinas a gás. Tese de doutorado, Universidade Federal de Itajubá.

Coelho, S. T., Velázquez, S. M. S. G., Martins, O. S., Abreu, F. C. (2006). A conversão da fonte renovável biogás em energia. Políticas públicas para a Energia: Desafios para o próximo quadriênio; Brasília.

Cormio C, Dicorato M, Minoia A, Trovato M. (2003). A regional energy planning methodology including RES and environmental constraints; Renewable and Sustainable Energy, pp. 99-130.

Cox, E. (1994). The Fuzzy Systems Handbook: a practitioner's guide to building, using, and maintaining fuzzy systems. Academic.

Cvansnick, J.C. (1995). Uma nova abordagem ao problema de construção de uma função de valor cardinal: MACBETH. Investigação Operacional; vol. 15, pp. 15-35.

Dutra, R. M., Szklo, A. S. (2008). Incentive policies for promoting wind power energy, vol. 33, pp. 65-76.

Edinger, R., Sanjay, K. (2000). Renewable Resources for Electric Power, Quorum Books.

Farret, F. A., Simoes, M. G. (2006). Integration of Alternative Sources of Energy. John Wiley \& Sons.

Haralambopoulos, D.A., Polatidis H. (2003) Renewable energy projects: structuring a multi-criteria group decision making framework. Renewable Energy, vol. 28 (6), pp. 961-73.

Hashem Nehrir, M. (2006) A Course on Alternative Energy Wind/PV/Fuel Cell Power Generation; in proceedings of the IEEE.
Liang, G., Wang, M. (1991). A Fuzzy Multi-criteria Decision Method for Facility Selection, International Journal of Production Research, vol. 29 (11), pp. 2313-1330.

Lora, E.E.S., Maddad, J. (2006). Geração Distribuída, Aspectos Tecnológicos, Ambientais e Institucionais. Ed. Interciência Ltda.

Pedrycz, W., Gomide, F. (1988). An introduction to fuzzy sets: analysis and design. Massachusetts: The MIT Press.

Programa de Incentivo a Fontes de Energia Alternativas; Lei 10.438/2002. Anais eletrônicos. Disponível em: http://www.mme.gov.br/programs_display.do?chn $=877$.

Saaty, T. L. (1991). Método de Análise Hierárquica. Makron Books do Brasil Editora Ltda. e Editora McGraw-Hill do Brasil, Rio de Janeiro/RJ.

Soares, C. Microturbines. Elsevier, pp. 271, 2007.

Shaw, I. S.; Simões, M. G. (1999). Controle e Modelagem Fuzzy. $1^{\circ}$. ed. São Paulo: Edgard Blücher Ltda.

Wedley, W.C., Ung Choo, E., Schoner, B. (2001). Magnitude adjustment for AHP benefit/cost ratios. European Journal of Operational Research, vol. 133, issue. 2, pp. 342-35. 

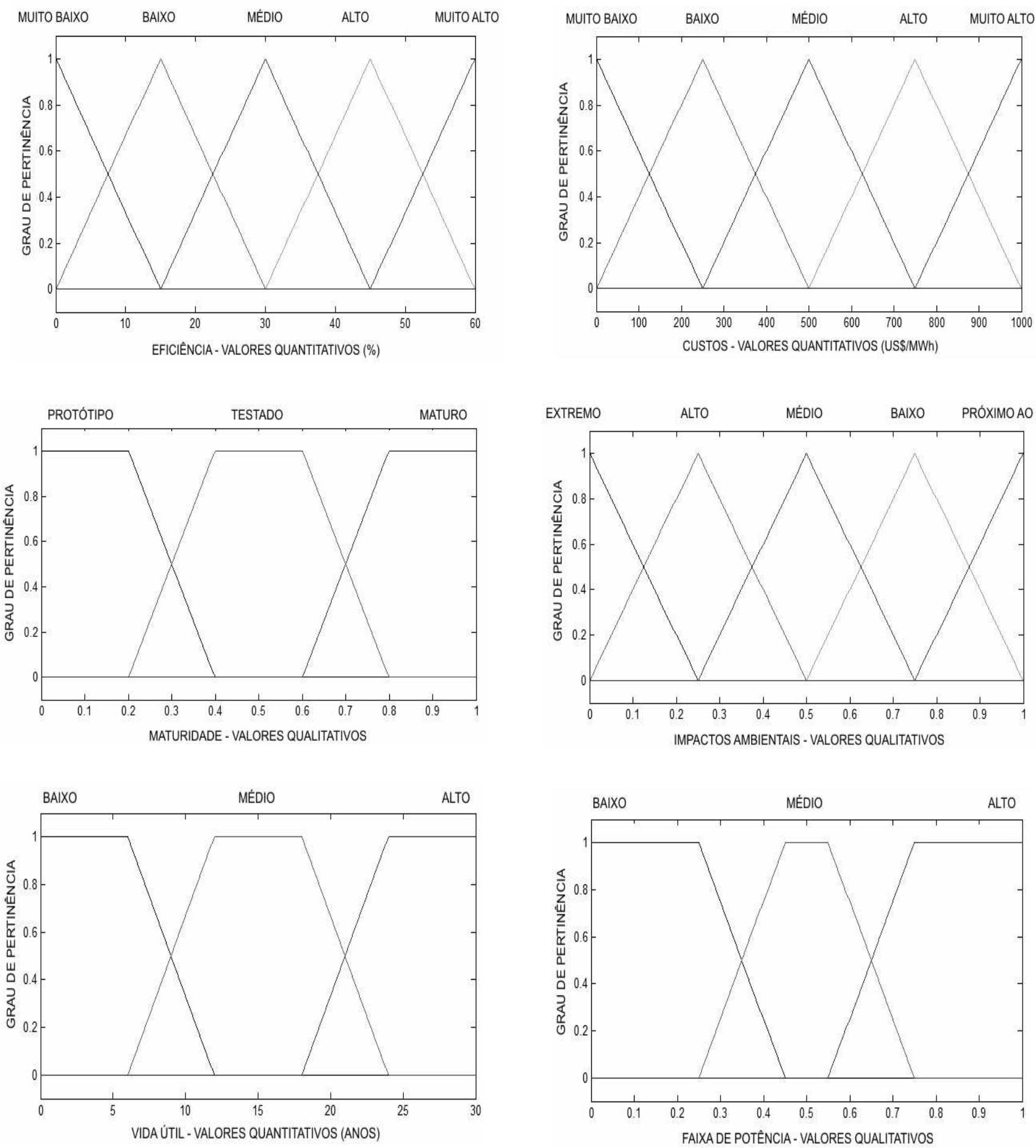

Figura 1: Conjuntos Fuzzy. 